PROCEEDINGS OF THE

AMERICAN MATHEMATICAL SOCIETY

Volume 133, Number 10, Pages 3097-3101

S 0002-9939(05)07878-0

Article electronically published on March 24, 2005

\title{
A CHARACTERIZATION OF THE PROJECTIVE LINE
}

\author{
B. REQUEJO AND JUAN B. SANCHO
}

(Communicated by Michael Stillman)

\begin{abstract}
Let $X$ be a set (with at least three different points) and let $G$ be a group of bijections of $X$. If the action of $G$ on $X$ satisfies three natural conditions, then $X$ admits a canonical structure of a projective line over a commutative field, such that $G$ is the group of all projective transformations of $X$.
\end{abstract}

\section{INTRODUCTION}

Given a commutative field $K$, let us recall that the projective line $\mathbb{P}_{1}$ over $K$ is defined to be the set of all 1-dimensional subspaces of some 2-dimensional vector space $V$ over $K$. A projective transformation is a bijection of $\mathbb{P}_{1}$ induced by some linear automorphism of $V$.

The purpose of this note is to prove the following group-theoretic characterization of the projective line.

Theorem 1.1. Let $G \times X \rightarrow X$ be an action of a group $G$ on a set $X$ (with at least three different points) satisfying the following conditions:

$\mathrm{A}_{1}$ Given three different points $x_{1}, x_{2}, x_{3} \in X$ and another three different points $y_{1}, y_{2}, y_{3} \in X$, there exists a unique transformation $g \in G$ such that $g\left(x_{i}\right)=$ $y_{i}$ for any $i=1,2,3$.

$\mathrm{A}_{2}$ Given three different points $p, x, y \in X$, there exists a unique transformation $g \in G$ such that $g(x)=y$ and $p$ is the unique fixed point of $g$.

B Let $g \in G$. If there exist two different points $x, y \in X$ such that $g(x)=y$ and $g(y)=x$, then $g$ is an involution: $g^{2}=I d$.

Then $X$ admits a natural structure of a projective line over a commutative field $K$, such that $G$ is the group of all projective transformations of $X$.

Axiom $\mathrm{A}_{1}$ implies that any transformation $g \in G$ with three fixed points is the identity map. We may think of axiom $\mathrm{A}_{2}$ as a degenerate case of $\mathrm{A}_{1}$ imagining $p$ as a double-counted point.

As a preliminary step in the proof of Theorem 1.1 we give a similar characterization of the affine line.

In the case of dimension $n>1$, there exist group-theoretic definitions of the affine and projective geometries in terms of the group of dilatations (see 2] and [3]).

Received by the editors December 1, 2003 and, in revised form, May 20, 2004.

2000 Mathematics Subject Classification. Primary 51A05.

(C)2005 American Mathematical Society Reverts to public domain 28 years from publication 


\section{Characterization of the affine line}

As is well known, there exist two elementary definitions for the affine space: the synthetic definition and the algebraic one. For dimension 1, only the algebraic definition makes sense. Let us recall it.

Let $K$ be a commutative field. An affine space is a set $\mathbb{A}$ endowed with an action $\mu: V \times \mathbb{A} \rightarrow \mathbb{A}$ of a $K$-vector space $V$ on $\mathbb{A}$. It is assumed that the action satisfies the following property: Given $p, q \in \mathbb{A}$, there exists a unique vector $v \in V$ such that $q=p+v$ (we denote $p+v=\mu(v, p)$ ). The dimension of $\mathbb{A}$ is, by definition, the dimension of the vector space $V$.

A bijection $\varphi: \mathbb{A} \rightarrow \mathbb{A}$ is said to be an affine transformation if there exists a (necessarily unique) $K$-linear automorphism $\phi: V \rightarrow V$ such that

$$
\varphi(p+v)=\varphi(p)+\phi(v)
$$

for any $p \in \mathbb{A}$ and $v \in V$.

Of course, an affine line $\mathbb{A}$ is an affine space of dimension 1 . Once we fix a point $p_{0}$ (the origin) in the affine line $\mathbb{A}$ and a vector $v \neq 0$ in $V$, we may write any point $p \in \mathbb{A}$ in the form $p=p_{0}+x v$ for some scalar $x \in K$; the scalar $x$ is said to be the affine coordinate of $p$. In terms of affine coordinates, any affine transformation $\varphi: \mathbb{A} \rightarrow \mathbb{A}$ may be written in the form

$$
\varphi(x)=a x+b \quad \text { for some } a, b \in K .
$$

Theorem 2.1. Let $G \times X \rightarrow X$ be an action of a group $G$ on a set $X$ satisfying the following conditions:

$\mathrm{C}_{1}$ Given two different points $x_{1}, x_{2} \in X$ and another two different points $y_{1}, y_{2} \in X$, there exists a unique transformation $g \in G$ such that $g\left(x_{i}\right)=y_{i}$ for any $i=1,2$.

$\mathrm{C}_{2}$ Given two different points $x, y \in X$, there exists a unique transformation $g \in G$, without fixed points, such that $g(x)=y$.

D For each point $x \in X$, the isotropy subgroup $H_{x}$ is commutative.

Then $X$ admits a natural structure of an affine line over a commutative field $K$ such that $G$ is the group of all affine transformations of $X$.

The proof of this theorem is a modification of the introduction of coordinates in the affine space used by Artin [1].

Under the hypotheses of the above theorem, let us introduce some terminology. A transformation $\tau \in G$ is said to be a translation if it has no fixed points or it is the identity. Given points $x, y \in X$, axiom $\mathrm{C}_{2}$ states that there exists a unique translation $\tau \in G$ such that $\tau(x)=y$.

A transformation $\sigma \in G$ with a fixed point $p_{0}$ is said to be a homothety with centre $p_{0}$. Axiom D states that the subgroup of all homotheties with centre $p_{0}$ is commutative.

Lemma 2.2. The set $T$ of all translations is a normal subgroup of $G$.

Proof. $T$ is a subgroup: Since the identity is a translation and the inverse of a translation is clearly a translation, we only need to show that the composition of two translations $\tau_{1} \tau_{2}$ is a translation, i.e., $\tau_{1} \tau_{2}$ has no fixed points or it is the identity. If $x$ is a fixed point of $\tau_{1} \tau_{2}$, then $\tau_{2}(x)=\tau_{1}^{-1}(x)$. By the uniqueness condition of $\mathrm{C}_{2}$, we obtain that $\tau_{2}=\tau_{1}^{-1}$; hence $\tau_{1} \tau_{2}=I d$.

It is immediate to check that $T$ is normal in $G$. 
Lemma 2.3. Let $\tau_{1}, \tau_{2}$ be translations different from $I d$. Given a point $p_{0} \in X$, there exists a unique homothety $\sigma$ with centre $p_{0}$ such that $\sigma \tau_{1} \sigma^{-1}=\tau_{2}$.

Proof. Let $p_{1}=\tau_{1}\left(p_{0}\right)$ and $p_{2}=\tau_{2}\left(p_{0}\right)$. By $\mathrm{C}_{1}$, there exists a unique homothety $\sigma$ with centre $p_{0}$ such that $\sigma\left(p_{1}\right)=p_{2}$. Both translations $\sigma \tau_{1} \sigma^{-1}$ and $\tau_{2}$ map $p_{0}$ to $p_{2}$; hence they coincide: $\sigma \tau_{1} \sigma^{-1}=\tau_{2}$. Conversely, if $\sigma$ is a homothety with centre $p_{0}$ such that $\sigma \tau_{1} \sigma^{-1}=\tau_{2}$, then $\left(\sigma \tau_{1} \sigma^{-1}\right)\left(p_{0}\right)=\tau_{2}\left(p_{0}\right)$; hence $\sigma\left(p_{1}\right)=p_{2}$.

Proposition 2.4. The group $T$ of all translations is commutative.

Proof. Let $\tau_{1}, \tau_{2}$ be translations different from $I d$. By Lemma 2.3 , there exists a homothety $\sigma$ such that

$$
\sigma\left(\tau_{2} \tau_{1} \tau_{2}^{-1}\right) \sigma^{-1}=\tau_{1}
$$

If $\sigma \tau_{2}$ has some fixed point (i.e., it is a homothety), then the uniqueness part of Lemma 2.3 used in the equality

$$
\left(\sigma \tau_{2}\right) \tau_{1}\left(\tau_{2}^{-1} \sigma^{-1}\right)=\tau_{1}
$$

implies that $\sigma \tau_{2}=I d$; hence $\sigma=\tau_{2}^{-1}$ has no fixed point, contradicting that $\sigma$ is a homothety. Therefore, $\sigma \tau_{2}$ has no fixed point (i.e., it is a translation); hence $\sigma$ is a translation. Since $\sigma$ is a homothety, we conclude that $\sigma=I d$ and then

$$
\tau_{2} \tau_{1} \tau_{2}^{-1}=\tau_{1}
$$

or, equivalently, $\tau_{2} \tau_{1}=\tau_{1} \tau_{2}$.

Since $T$ is a normal subgroup of $G$, we may consider the action of $G$ on $T$ by conjugation. Let us consider the following ring with unity:

$$
K:=\operatorname{End}_{G}(T)
$$

Each element $\lambda \in K$ is an endomorphism of $T$ which commutes with the action of $G$. The product operation in $K$ is the composition of endomorphisms. Let us show that $K$ is a field.

Proposition 2.5. Let $\lambda: T \rightarrow T$ be an endomorphism which commutes with the action of $G$. Then $\lambda$ is the zero map or it is a bijection.

As a consequence, it follows that $K$ is a field.

Proof. By Lemma 2.3, $G$ acts transitively on $T-\{0\}$ (additive notation: 0 represents the identity translation). Since $\lambda$ commutes with the action of $G$, we conclude that $\operatorname{ker} \lambda=0$ or $\operatorname{ker} \lambda=T$, and $\operatorname{Im} \lambda=T$ or $\operatorname{Im} \lambda=0$.

Now we shall show that the product in $K$ is commutative.

Lemma 2.6. Fix a point $p_{0} \in X$. Given a transformation $g \in G$, there exists a unique translation $\tau$ and a unique homothety $\sigma$ with centre $p_{0}$ such that $g=\sigma \tau$.

Proof. Let $p_{1} \in X$ such that $g\left(p_{1}\right)=p_{0}$ and let $\tau$ be the unique translation such that $\tau\left(p_{1}\right)=p_{0}$. Then $\sigma:=g \tau^{-1}$ is the desired homothety with centre $p_{0}$.

Let $H$ be the subgroup of $G$ of all homotheties with centre $p_{0} . H$ is a commutative subgroup by axiom $\mathrm{D}$. The above lemma implies that the natural morphism

$$
H \longrightarrow G / T
$$

is an isomorphism. Since the action by conjugation $G \rightarrow \operatorname{End}(T)$ factors through the quotient $G / T=H$ and $H$ is commutative, we conclude that any element $g \in G$ defines, acting by conjugation on $T$, a scalar $\lambda_{g} \in K=\operatorname{End}_{G}(T)$. 
Proposition 2.7. The field $K=\operatorname{End}_{G}(T)$ is commutative, and $T$ is a $K$-vector space of dimension 1.

Proof. Given two non-zero translations $\tau_{1}, \tau_{2} \in T$, there exists a homothety $\sigma \in H$ such that $\sigma \tau_{1} \sigma^{-1}=\tau_{2}$ (see Lemma 2.3); ; hence $\lambda_{\sigma} \cdot \tau_{1}=\tau_{2}$. This fact implies that $T$ has dimension 1 and that the morphism $H \rightarrow K^{*}, \sigma \mapsto \lambda_{\sigma}$, is surjective. Since $H$ is commutative (axiom $\mathrm{D}$ ), we conclude that $K$ is a commutative field.

Proof of Theorem 2.1. Since $T$ is a $K$-vector space of dimension 1, $X$ inherits the structure of an affine line via the action of $T$ on $X$.

Given $\sigma \in G$, for any translation $\tau \in T$ we have $\sigma \tau \sigma^{-1}=\lambda_{\sigma} \cdot \tau$; hence $\sigma \tau=$ $\left(\lambda_{\sigma} \cdot \tau\right) \sigma$. Rewriting this last equality using the additive notation $\tau(p)=p+v$, we obtain that

$$
\sigma(p+v)=\sigma(p)+\lambda_{\sigma} v
$$

hence $\sigma$ is an affine transformation of $X$. Conversely, using axiom $\mathrm{C}_{1}$, it is immediate that any affine transformation of $X$ coincides with some element of $G$.

\section{Proof OF the MAIN RESUlt}

With the hypotheses of Theorem 1.1, let us fix a point $p_{\infty} \in X$. Let

$$
X^{\prime}=X-\left\{p_{\infty}\right\}, \quad G^{\prime}=\left\{g \in G: g\left(p_{\infty}\right)=p_{\infty}\right\} .
$$

Lemma 3.1. The action of $G^{\prime}$ on $X^{\prime}$ satisfies all the conditions of Theorem 2.1.

Proof. It is clear that axioms $\mathrm{A}_{1}$ and $\mathrm{A}_{2}$ imply $\mathrm{C}_{1}$ and $\mathrm{C}_{2}$.

To prove condition $\mathrm{D}$, given any point $p_{0} \in X^{\prime}$, let us consider a transformation $g \in G$ such that $g\left(p_{0}\right)=p_{\infty}$ and $g\left(p_{\infty}\right)=p_{0}$. By axiom B, we have that $g^{2}=I d$. Let

$$
H_{p_{0}}=\left\{\sigma \in G^{\prime}: \sigma\left(p_{0}\right)=p_{0}\right\}=\left\{\sigma \in G: \sigma\left(p_{0}\right)=p_{0} \text { and } \sigma\left(p_{\infty}\right)=p_{\infty}\right\}
$$

be the isotropy subgroup (in $G^{\prime}$ ) of $p_{0}$. By conjugation with $g$, we obtain an automorphism of groups

$$
\varphi: H_{p_{0}} \longrightarrow H_{p_{0}}, \quad \sigma \mapsto g \sigma g .
$$

For any $\sigma \in H_{p_{0}}$, we have that $g\left(\sigma\left(p_{0}\right)\right)=p_{\infty}$ and $g\left(\sigma\left(p_{\infty}\right)\right)=p_{0}$; hence $g \sigma g \sigma=I d$ by axiom B. This implies that $\varphi(\sigma)=\sigma^{-1}$ and, since $\varphi$ is an automorphism of groups, we conclude that the isotropy group $H_{p_{0}}$ is commutative.

Proof of Theorem 1.1. Applying Theorem 2.1 to the action $G^{\prime} \times X^{\prime} \rightarrow X^{\prime}$, we obtain that $X^{\prime}$ is an affine line over a commutative field $K$, and $G^{\prime}$ is the group of all affine transformations of $X^{\prime}$. Taking an affine coordinate on $X^{\prime}$, we may write $X=K \cup\{\infty\}$, i.e., $X$ is a projective line over the field $K$. $X$.

Now we have to prove that $G$ is the group of all projective transformations of

Let $g \in G$ be the unique transformation such that $g(0)=\infty, g(1)=1$ and $g(\infty)=0$ (note that $g$ is an involution by axiom B). Now we shall prove that $g(x)=x^{-1}$ for any $x \in X$. Let $\sigma_{\lambda} \in G^{\prime}$ be the homothety with centre 0 and ratio $\lambda$, i.e., $\sigma_{\lambda}(x)=\lambda x$. The same argument used in the proof of Lemma 3.1 gives that $g \sigma_{\lambda} g \sigma_{\lambda}=I d$; hence $g \sigma_{\lambda} g=\sigma_{\lambda^{-1}}$. On the other hand, $g \sigma_{\lambda} g=\sigma_{g(\lambda)}$ because $g \sigma_{\lambda} g$ is a homothety with centre 0 and $\left(g \sigma_{\lambda} g\right)(1)=g(\lambda)$. In conclusion, it follows that $g(\lambda)=\lambda^{-1}$. 
Since $G$ contains the transformation $x \mapsto a x+b$ (because $G$ contains the group $G^{\prime}$ of the affine transformations) and it also contains the transformation $g: x \mapsto$ $x^{-1}$, we conclude that $G$ contains all the projective transformations $x \mapsto \frac{a x+b}{c x+d}$. Conversely, using the uniqueness part of axiom $\mathrm{A}_{1}$, it is immediate that any element of $G$ is a projective transformation.

\section{FURTHER COMMENTS}

The projective line $\mathbb{P}_{1}$ over a commutative field $K$ is usually defined as the set of all 1 -dimensional subspaces of some 2 -dimensional vector space $V$ over $K$. This definition is not satisfactory since it presents the projective line as a set without any structure. This makes obscure the notion of an automorphism of $\mathbb{P}_{1}$. An automorphism of $\mathbb{P}_{1}$ should be a bijection preserving the "structure", but which structure? If $\mathbb{P}_{1}$ were a mere set, then its automorphisms should be bijections of this set; then we ought to define the notion of projective transformation as an arbitrary bijection!

Of course, the question about the structure of $\mathbb{P}_{1}$ is illuminated by modern Algebraic Geometry: $\mathbb{P}_{1}$ has the structure of a scheme. Scheme automorphisms of $\mathbb{P}_{1}$ are just projective transformations in the sense of Staudt (= bijections of $\mathbb{P}_{1}$ induced by semilinear automorphisms of $V$ ), while ordinary projective transformations are automorphisms of $\mathbb{P}_{1}$ as a $K$-scheme, i.e., scheme automorphisms of $\mathbb{P}_{1}$ which define the identity on $\mathrm{H}^{0}\left(\mathbb{P}_{1}, \mathcal{O}_{\mathbb{P}_{1}}\right)=K$. A conceptually rigorous foundation of Projective Geometry may be considered as a first great result of Grothendieck's theory of schemes.

Our characterization allows us to give an alternative "structural" definition of the projective line, in the spirit of Klein's Erlangen Program: A projective line is a pair $(X, G)$ where $X$ is a set and $G$ is a subgroup of $\operatorname{Bij}(X)$ satisfying the conditions of Theorem 1.1. The automorphisms of this structure are the bijections $\varphi: X \rightarrow X$ preserving the subgroup $G$, i.e., $\varphi G \varphi^{-1}=G$. These automorphisms correspond to Staudt transformations in the conventional definition of $\mathbb{P}_{1}$, while the elements of $G$ correspond to ordinary projective transformations.

\section{REFERENCES}

[1] E. Artin, "Geometric Algebra", Interscience, New York, 1957. MR0082463 (18:553e)

[2] A.D. Gottlier and J. Lipman, Group-theoretic axioms for projective geometry, Canad. J. Math. 43 (1) (1991), 89-107. MR1108915 (92g:51002)

[3] J. Lipman, Definition of affine geometry by a group of transformations, Canad. Math. Bull. 4 (1961), 265-278. MR0131194 (24:A1047)

Departamento de Matemáticas, Universidad de Extremadura, 06071 Badajoz, Spain

E-mail address: brequejo@unex.es

Departamento de Matemáticas, Universidad de Extremadura, 06071 Badajoz, Spain

E-mail address: jsancho@unex.es 